\title{
EDI70RIAL
}

\section{A Little Effort}

Little drops of water, little grains of sand Make the mighty ocean and the land pleasant Thus the little minutes, little though they be

Make the might ages of eternity

\section{E. C. Brewer}

The value of little can hardly be exaggerated as expressed in the inspirational lines above. Confucius confirmed Brewer when he said "A journey of a thousand miles begins with a single step". Nevertheless, while we are all set to bring forth the maiden issue of Academic Voices, we are not engrossed by any vanity, but a significant realization comes to us that a little effort we have made and have a long way to cover.

The journal in your hand reflects the academic wealth that the campus possesses. Although the campus published some magazines or mouthpieces such as Himal, Smarika, Bibechana etc., in the past, it is the first time that Thakur Ram has brought an academic journal into publication. Additionally, the journal carries significance in that it is peer-reviewed. Previously, though a few teachers had opportunities for their professional growth via publication, majority of them suffered owing to lack of publication culture in the campus resulting into fossilization of their professional caliber. Now as the journal has given them a space to share their experiences and reflections into paper, atmosphere of the campus feels to be radically changed. We have observed changes in the attitude of the teachers towards publication and they have understood that writing is not a piece of cake but they have to pass through different stages from submission of article to its publication. This is a big achievement, we belive.

'No great work can be done without sacrifice', said Vivekanand. And it applies to this journal as well. We are very sorry to say that we have spent almost a year to bring it to publication. However, we do not blame anybody for it-neither authors, nor reviewers. This happens when an innovative venture is undertaken and so happened here. Encouraging teachers to submit articles abiding by the submission guidelines, selecting experts in the concerned field for the review process, suggesting authors to modify manuscripts in accordance with the reviewers' comments, verifying the resubmitted versions of the manuscripts, editing and proof-reading them meticulously took a lot of time, two times more than the scheduled. However, we have learnt a lot and so have authors, we hope. We are very thankful to the authors who repeatedly revised their manuscripts in accordance with comments given by the 
reviewers and editorial board. Likewise, words fall short to express our gratefulness to the reviewers who gave time and took pain in the review process to uplift the quality of the journal. The journal would not have come in the present form without their assistance. We are very sorry that we could not incorporate a couple articles as they did not meet the academic rigor required by the journal.

'Academic Voices' is multidisciplinary in nature which includes contributions from various disciplines of science, education, social sciences etc. Whearas the article by Vishwanath Prasad presents recent information about position of cyanobacteria in the evolution of higher plants. Umesh Prasad Srivastava describes the potential impact of transgenic plants in developing countries in his review article. Similalry,Pramod Kumar Kushwaha's research based article deals with cephalic indices of some birds in relation to their feeding habits.

In the articles concerning with English education, Jai Prakash Singh, Kedar Prasad Sah, Renu Singh concentrate on some of the ELT issues such as effectiveness of Total Physical Response, reflections on Meaning into Words Intermediate and controversies in grammar teaching respectively.

In the articles related to Math education, Dhananjay Kumar Tiwari raises an issue of fundamental forces and unified field theory, Bidyannad Prasad describes category of fuzzy sets and Kripa Sindhu Prasad highlights learning mathematics by discovery.

Whereas Satyadev Prasad Yadav and Matrika Prasad Koirala have pondered over democratization and existentialism in education, Bhawani Prasad Mainali and Tara Bahadur Thapa have advocated to adopt Critical Thinking(CT) and Total Quality Management(TQM) for quality education in Nepal respectively.

On a different note, Om Prasad Baral has tried to penetrate into the issue of HIV/AIDS epidemic, Keshav Kumar Yadav analyses changing population characteristics of Parsa. And Manju Shrestha has spent words to justify the relevance of women reservations in her article.

In a constructively critical article, Sajan Kumar Karn, taking a case of Thakur Ram Multiple Campus, evaluates Nepalese academia, against the backdrop of recent paradigm shifts in education around the globe and also proposes for some radical changes in order to save it from deadly future that it has envisaged.

Last but far from the least, Abdul Quaiyum, in his article, highlights the features of Mathematics in Contexts (MiC) and its relevance in the Nepalese Math Education.

We are very optimistic that you enjoy reading the papers in this volume but we will be happier if you could share your thoughts and comments to us via academicvoices2010@gmail.com. 